\title{
A Method for the Identification of Noisy Regions in Normalized Iris Images
}

\author{
Hugo Proença and Luís A. Alexandre \\ Dep. Informatics, IT - Networks and Multimedia Group \\ Universidade da Beira Interior, Covilhã, Portugal \\ Email: \{hugomcp,lfbaa\}@di.ubi.pt
}

\begin{abstract}
In this paper we propose a new method for the identification of noisy regions in normalized iris images. Starting from a normalized and dimensionless iris image in the polar coordinate system, our goal consists in the classification of every pixel as "noise" or "not noise". This classification could be helpful in the posterior feature extraction or feature comparison stages regarding the construction of biometric iris signatures more robust to noise. We propose the extraction of 8 well known features for each pixel of the images followed by the classification through a neural network.
\end{abstract}

Keywords: noise identification, classification, neural networks, iris recognition, biometrics.

\section{Introduction}

The use of biometric systems has been increasingly encouraged by both government and private entities in order to replace or improve traditional security systems. Moreover, the dynamic conditions of the environments where iris recognition systems are functioning propitiate the capture of very heterogeneous images, regarding focus, brightness, contrast, reflections or iris obstructions (eyelids, eyelashes, glasses, ...). These noise factors led to a substantial increment of the false rejections rate, a problem commonly identified by several authors (e.g. [18], [11] and [17]).

Our aim consists in the identification of noisy regions in the iris images and posterior construction of a binary map where the noisy pixels are marked as opposed to non-noisy pixels. This map can be useful in the posterior stages of the iris recognition, namely the feature extraction and comparison stages.

We have analyzed the public and freely available iris databases ([13], [3], [6], [16], and [14]) and choused the
UBIRIS [14] due to the high heterogeneity of the images regarding focus, reflections, contrast and lighting conditions.

Observations led us to identify five different types of noise (information other than iris) that can corrupt the iris regions:

Eyelids The biological function and natural movement of the eyelids can obstruct relevant portions of the iris, specially on the upper and lower parts. Number 1 from the figures $1 \mathrm{a}$ and $1 \mathrm{~b}$ illustrates the obstruction of the iris by eyelids respectively in the captured and in the normalized and dimensionless image in the polar coordinate system [2].

Eyelashes Eyelashes can obstruct portions of the iris in two distinct forms as they appear isolated or grouped. If one eyelash is isolated, it appears as a very thin and darker line in the iris region. The existence of multiple eyelashes in the iris regions generates a uniform darker region. Number 2 in the figures $1 \mathrm{a}$ and $1 \mathrm{~b}$ illustrates examples of eyelashes obstructions.

Pupil When the segmentation of the pupil is not accurate, some portions of the pupil will be considered as iris parts. Those areas appear at the upper part of the normalized iris image (number 5 from figure $1 b$ ).

Strong Reflections We consider areas with "strong reflections" those that correspond to reflections of light sources directly pointed at the iris. These areas have intensity values close to the maximum (number 4 of images $1 \mathrm{a}$ and $1 \mathrm{~b}$ ).

Weak Reflections This type of reflections correspond to reflected information from the environment where the user is located and is looking at. As illustrated by the number 3 from the figures $1 \mathrm{a}$ and $1 \mathrm{~b}$, these reflections have lower intensity values than the previous ones and can correspond to a wide range of objects that the user is surrounded by.

The remaining sections of this paper are organized as follows: in section 2 we overview several methods for the 


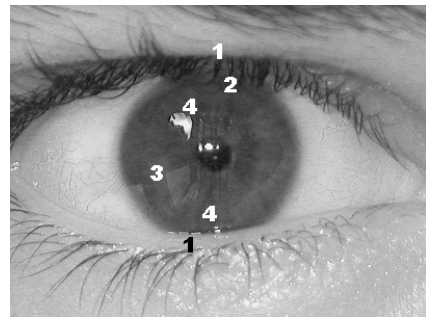

(a) Captured iris image

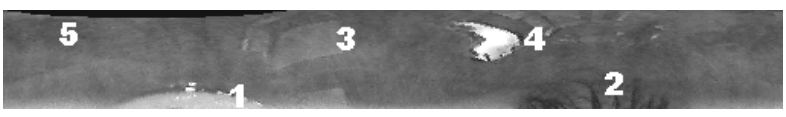

(b) Segmented and normalized iris image

\section{Figure 1. Types of noise in an iris image}

identification of noise in iris images. Section 3 describes the proposed classification methodology and in section 4 we discuss and compare the obtained results. Finally, section 5 lists the conclusions.

\section{Related Work}

In order to avoid the problems originated by noisy information, some authors (e.g. [2]) propose the utilization of portions of the iris, those where noise factors are less probable.

In [10] the authors propose an iris image enhancement by means of local histogram equalization and the removal of the high-frequency noise through Gaussian low-pass filtering.

Motivated by the observed difference between the standard deviation of the intensity values within small windows from noisy and noise-free regions, authors from [12] and [4] propose the computation of the standard deviations within small $(3 \times 3$ and $5 \times 5$ pixels $)$ windows. If the value is superior to a threshold, the central pixel of the window is considered noise.

Wildes [18] proposes the equalization of the histogram of the whole image and the localization of the inferior and superior eyelids by means of an edge detection algorithm followed by the linear Hough transform.

In [9] a global measure of the quality of the captured image is proposed, based on the analysis of its frequency distribution. The authors claim that noise-free irises have relatively uniform distribution, as opposed to poor focused or eyelid or eyelashes obstructed images. The authors did not identify eyelashes or eyelids, having however concluded that a substantial part of the observed false rejections were due to the eyelid and eyelash obstruction (57.7\%) and inaccurate iris segmentation $(21.1 \%)$.

The authors of [8] propose the classification of the noisy regions directly in the captured image. They identified the separable (isolated) eyelashes through the energy of the convolution of the image with a bank of Gabor filters. The values lower than a threshold correspond to the noisy regions. Multiple eyelashes were identified through the computation of the standard deviation within small regions of the image. Reflections were classified as "strong" (identified with a simple threshold) and "weak". The latter correspond to transitions between the strong reflections and the noise-free areas and are identified through an iterative algorithm that expands the strong reflections areas.

Motivated by the problem of the high false rejection rates, the authors of [17] propose the use of an edge detector followed by the linear Hough transform to detect eyelids and eyelashes. This approach was also proposed with minor variants in [7].

Based on the analysis of the energy resultant from the convolution between the image and a group of Mexican-Hat Wavelets at three different scales, the authors of [1] propose both local and global image quality measures. From our viewpoint, the database used was not adequate for the effective test of the methodology, since it contains almost no reflections.

The purpose of the authors of [5] was the identification of four distinct types of noise: eyelashes, eyelids, reflections and pupil. The basic idea is that there's always some type of edge between the noisy and the noise-free areas. Those edges were identified through an illumination invariant measure (phase congruency).

The analysis of the above described methods allowed us to divide them as:

- Global versus local methodologies. Global methods seek for the classification of the whole image, as "poor focused" or "obstructed iris". Local methods try to classify each pixel of the iris providing additional information about each region of the image.

- Analysis of the captured versus the normalized iris image. Some authors propose the noise identification (usually eyelids and eyelashes) before the normalization of the segmented image. We think that the utilization of the whole image, containing more information than the iris regions will obviously decrease the method's accuracy and increase its computational complexity.

For the comparison between our methodology and other proposals, we selected the methods from [5] and [1] since they provide local quality measures, they operate in the normalized iris image and their authors claimed invariance to illumination changes. 


\section{Table 1. Noise identification errors from tested measures}

\begin{tabular}{|l|c|}
\hline Method & Error Rate (\%) \\
\hline ASM & $30.21 \pm 0.0652$ \\
\hline Entropy & $11.17 \pm 0.0447$ \\
\hline Contrast & $40.18 \pm 0.0696$ \\
\hline Energy & $30.10 \pm 0.0651$ \\
\hline Inertia & $30.78 \pm 0.0655$ \\
\hline Phase Congruency ([5] methodology) & $14.61 \pm 0.0501$ \\
\hline$[1]$ methodology & $20.83 \pm 0.0577$ \\
\hline Our methodology & $2.74 \pm 0.0232$ \\
\hline
\end{tabular}

\section{Proposed Methodology}

In this section we will describe the proposed methodology for the identification of noisy regions. We start by the extraction of 8 well known features followed by the classification through a fully connected feed-forward neural network with one hidden layer.

\subsection{Feature Extraction}

Centered at each pixel, we computed five commonly used statistical measures in small image windows, as described in [15]: Angular Second Moment (ASM), Entropy, Contrast, Energy and Inertia. We made the classification through simple thresholds and achieved the results presented in the upper 5 rows of table 1 . The two bottom rows correspond to the obtained error rates respectively by the methodologies proposed by [5] and [1]. These results correspond to a 99\% confidence interval from the classification of 100 segmented, normalized and manually classified iris images from the UBIRIS database, each of them with fixed dimensions of $512 \times 64$ (width $\times$ height $)$ pixels. Experiments led us to choose $7 \times 7$ windows for the computation of the statistical measures.

In spite of the distinct error rates from each measure, we observed that each of the them emphasizes different types of noise. Based on this fact, we combined all these measures and the proposed by [5] in the following classification process.

In order to achieve spatial relationship between image regions, we considered the position of each pixel in the normalized image as features. Thus, for each image pixel, we computed a 8-dimensional feature vector: (row, column, entropy, ASM, energy, contrast, inertia, phase congruency).

\subsection{Classification}

In the classification stage we applied a fully connected feed-forward neural network with 1 hidden layer. The input and output layers have fixed number of neurons, respectively 8 neurons for the input and 2 neurons for the output layer. As discussed in section 4, we made several experiments varying the number of neurons on the hidden layer and the maximum allowable error in the learning process to achieve the optimization of the neural network's accuracy. Figure 2 contains the obtained classification map for the identified noisy regions from figure $1 \mathrm{~b}$. The dark regions correspond to noisy information and the white to iris regions.

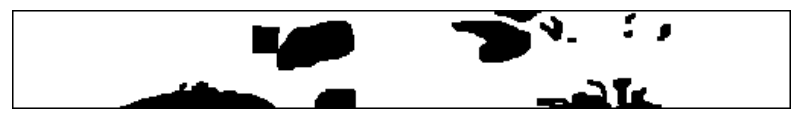

Figure 2. Identified noisy regions from figure 1b.

\section{Experiments and discussion}

In the same data set described in section 3.1, we randomly selected 20000 noisy and 20000 noise-free pixels, which represent just $0.12 \%$ of the whole data set. We proceed to the feature extraction process described in section 3.1 and used this data as the neural network learning set. In order to optimize the results, we evaluated the accuracy of the neural network varying each of the following parameters:

Number of neurons in the hidden layer Prior studies have demonstrated that this number has a strong influence on the neural network ability to optimally separate the data. Based on this, we varied the number of neurons in the hidden layer and repeated the learning process.

\section{Maximum allowable error to stop the learning stage}

This value defines the desirable average error of the neural network when classifying the training data set. It is important for overcoming the possibility of the excessive specialization of the network on the training data.

Figure 3 illustrates the obtained error rates in the test data. The presented values correspond to the average errors obtained after 10 repetitions of the learning process for each configuration. The "X" and "Y" axis respectively correspond to the number of neurons in the hidden layer and 


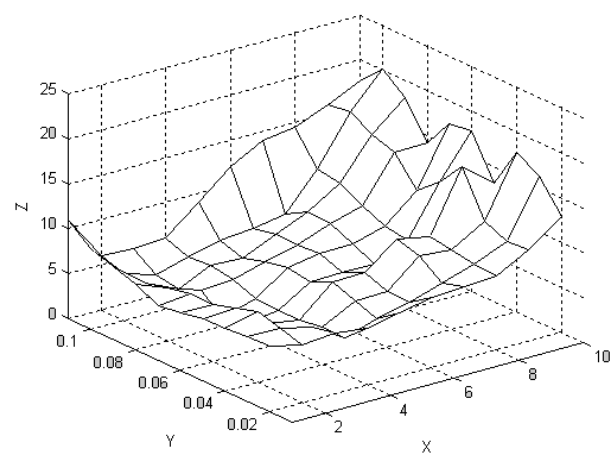

\section{Figure 3. Error rates of the neural network in the test data}

the maximum allowable error in the learning stage. The "Z" axis contains the obtained error rates. The best results were obtained with the 8:5:2 topology and the maximum allowable error in the learning stage of $0.03(3 \%)$. With this configuration the neural network is able to correctly classify $97.26 \% \pm 0.0232 \%$ of the test data, with false positives and false negatives rates of respectively $2.85 \% \pm 0.0236 \%$ and $1.78 \% \pm 0.0188 \%$ in a confidence interval of $99 \%$.

As can be seen by the comparison with the bottom rows from table 1 , the obtained error rates were lower than those obtained by [5] and [1] methodologies in the UBIRIS database. However, it must be noted that the last one was not specially thought for the identification of reflections and this fact significantly deteriorates its results.

\section{Conclusions}

Here we presented an overview of the most common approaches for the identification of noisy data in iris images and proposed a new methodology based on the extraction of 5 well known statistical image features together with the phase congruency and the pixel position. In the classification stage we used a fully connected feed-forward neural network with 1 hidden layer. Experiments led us to conclude that our method has a much better performance (only $2.74 \%$ error) than the other methods used in the comparison. This increase in performance is obtained at the cost of an increase in the computational effort.

\section{References}

[1] Y. Chen, S. C. Dass, and A. K. Jain. Localized iris image quality using 2-D wavelets. In Proceedings of the 2006 International Conference on Biometric, pages 373-381, Hong Kong, 2006.

[2] J. G. Daugman. High confidence visual recognition of persons by a test of statistical independence. IEEE Transactions on Pattern Analysis and Machine Intelligence, Vol. 25, No. 11, pages 1148-1161, November 1993.

[3] M. Dobes and L. Machala. UPOL iris image database, 2004. http://phoenix.inf.upol.cz/iris/

[4] Y. Du, R. Ives, D. Etter, T. Welch, and C. Chang. A new approach to iris pattern recognition. In Proceedings of the SPIE European Symposium on Optics/Photonics in Defence and Security, vol. 5612, pages 104-116, October 2004.

[5] J. Huang, Y. Wang, T. Tan, and J. Cui. A new iris segmentation method for recognition. In Proceedings of the 17th International Conference on Pattern Recognition (ICPRO4), vol. 3, pages 23-26, 2004.

[6] Institute of Automation, Chinese Academy of Sciences. CASIA iris image database, 2004. http: / / www.sinobiometrics.com

[7] R. Ives, A. Guindry, and D. Etter. Iris recognition using histogram analysis. In Proceedings of the 38th Asilomar Conference on Signals, Systems and Computers, pages 562 566, 2004.

[8] W.-K. Kong and D. Zhang. Detecting eyelash and reflection for accurate iris segmentation. International Journal of Pattern Recognition and Artificial Intelligence, vol. 17, no. 6, pages 1025-1034, 2003.

[9] L. Ma, T. Tan, Y. Wang, and D. Zhang. Personal identification based on iris texture analysis. IEEE Transactions on Pattern Analysis and Machine Intelligence, vol. 25, no. 12, pages 2519-2533, December 2003.

[10] L. Ma, Y. Wang, and T. Tan. Iris recognition using circular symmetric filters. In Proceedings of the 25th International Conference on Pattern Recognition (ICPRO2), vol. 2, pages 414-417, 2002.

[11] L. Ma, Y. Wang, and D. Zhang. Efficient iris recognition by characterizing key local variations. IEEE Transactions on Image Processing, vol. 13, no. 6, pages 739-750, June 2004.

[12] K. Nam, K. Yoon, J. Bark, and W. Yang. A feature extraction method for binary iris code construction. In Proceedings of the 2nd International Conference on Information Technology for Application, pages 284-288, 2004.

[13] N.Ritter and C. Barry. Database of 120 grayscale eye images, Lions Eye Institute, 2004.

[14] H. Proença and L. A. Alexandre. UBIRIS: A noisy iris image database. In 13th International Conference on Image Analysis and Processing (ICIAP2005), pages 970-977, September 2005. http: //iris.di.ubi.pt

[15] T. Randen and J. H. Husøy. Filtering for texture classification: a comparative study. IEEE Transactions on Pattern Analysis and Machine Inteligence, vol. 21, no. 4, pages 291310, 1999.

[16] University of Bath. University of Bath iris image database, 2004. www. bath.ac.uk/elec-eng/pages/sipg/

[17] M. Vatsa, R. Singh, and A. Noore. Reducing the false rejection rate of iris recognition using textural and topological features. International Journal of Signal Processing, vol. 2, no. 1, pages 66-72, 2005.

[18] R. P. Wildes. Iris recognition: an emerging biometric technology. In Proceedings of the IEEE, vol. 85, no.9, pages 1348-1363, U.S.A., September 1997. 